\title{
Experimental studies of light-wave phase shift by polymer dispersed liquid crystal films
}

\author{
S.M. Dmitriev ${ }^{2}$, V.P. Dick ${ }^{1}$, N.N. Kostyuk ${ }^{2}$, T.A. Dick ${ }^{2}$, V.A. Loiko ${ }^{1}$ \\ ${ }^{1}$ B.I. Stepanov Institute of Physics, NAS of Belarus, \\ 68, Nezalezhnasti Ave., Minsk, 220072, Belarus \\ ${ }^{2}$ Belarusian State University, \\ 4, Nezalezhnasti Ave., Minsk, 220030, Belarus
}

\begin{abstract}
Polymermethacrylate (PMMA) and polyvinyl butyral (PVB) thin films with the dispersed liquid crystal droplets are prepared by the described technique. The measurements of light wave polarization-independent phase shift induced by the electric field are displayed. It was been shown that the phase shift in polymethylmethacrylate films can approach five radians at the applied electric field about $10 \mathrm{~V} / \mu \mathrm{m}$.
\end{abstract}

Keywords: polymer dispersed liquid crystal, transmission, scattering, phase shift.

Manuscript received 02.02.10; accepted for publication 25.03.10; published online 30.04.10.

\section{Introduction}

The polymer dispersed liquid crystal (PDLC) films are currently under thorough examination [1-10]. The interest in such films arises from the fact that they possess a number of properties coming from their design features that may be important from the viewpoint of practical application. For example, they have a high mechanical strength and can be rather easily made in the form of large-size screens. They can be made in the form of flexible films and can be applied to arbitrary-shaped surface. Apart from this, PDLC films enable to skip polarizers making the design simpler and the image brighter.

A PDLC film is a film based on polymer with LC droplets, the size of which can normally range from fractions of micrometers up to several micrometers. Owing to the difference of polymer and LC refraction indexes such a film light-scattering. The light-scattering factor can change with the change of molecular orientation in LC-droplets due to applied electric field [11-14]. This property of PDLC films makes it possible to use them for controlling the optical radiation intensity.

Beside the possibility to control the light-scattering factor, PDLC films possess another important property they can control the phase of radiation that passes through the films $[2-5,6-10,12]$. When designing new optoelectronic devices, this property turns out more significant than the capability to control the optical radiation intensity.
This paper describes experimental researches of optical properties inherent to PDLC films. At the same time, special attention has been paid to their capability to control the light wave phase. With this purpose, sufficiently transparent weak-like-scattering PDLC films have been examined.

The technique of making the samples and method to measure transmission and phase shift are described in Sections 2 and 3. The analysis of the obtained results is presented in Section 4.

\section{Technique for making the samples}

To obtain LC dispersions, the authors used the method of phase separation induced by the evaporation of the dissolvent $[11,13]$. In the experiments, the authors used cyanobiphenyl-based liquid crystal (VIN-9). Polymethylmethacrylate (PMMA) and polyvinylbutyral (PVB) have specially been synthesized for this purpose and chosen as polymer matrix.

Some physical characteristics of the materials used by the authors are presented in Table 1 .

Table 1. Physical characteristics of the liquid crystal and polymer matrixes.

\begin{tabular}{|l|l|l|l|}
\hline Matrix & VIN-9 & PMMA & PVB \\
\hline Refractive index $n_{D}{ }^{20}$ & $\begin{array}{l}1.6804\left(n_{\|}\right) \\
1.5109\left(n_{\perp}\right)\end{array}$ & 1.492 & 1.485 \\
\hline Density at $20{ }^{\circ} \mathrm{C}, \mathrm{g} / \mathrm{cm}^{3}$ & 1.05 & 1.2 & 1.1 \\
\hline $\begin{array}{l}\text { Glass transition } \\
\text { temperature, }{ }^{\circ} \mathrm{C}\end{array}$ & - & 115 & 57 \\
\hline
\end{tabular}


The experimental samples were produced using polymer binder solubilization in toluene, acetone, ethyl acetate, and benzole. Surface active agents (SAA) and then liquid crystal VIN-9 were introduced to the obtained solutions. Palmic and oleic acids were used as anionogenic SAAs. Octadecylamine was used as cationactive SAA. As non-ionogenic SAAs, the authors used alkynophenol polyoethylated ethers (commercial title OP-10) in amount of 0.1-0.2 wt.\%. After that, a thin layer of the solution was deposited onto glass with a transparent conductive coating (indium oxide thin film). After the solution was fully evaporated under room conditions, the glass with a thin layer of dispersed liquid crystal formed on it was covered with the second glass with transparent current-conductive coating.

It should be noted that in the process of solution the evaporation time depended on the solution layer thickness applied to this glass. For thin films, it took less time as compared to the thick ones.

Therefore, the morphology of making films (particularly, LC droplets concentration and size) was governed by both the ratio LC/polymer in source solution and the thickness of formed PDLC films.

In the process of making the films, the developers comprised three types of solutions which differed in the weight ratio LC/PMMA: type A formed from $55 \% \mathrm{LC}$ and $45 \%$ PMMA, type B formed from $65 \% \mathrm{LC}$ and $35 \%$ PMMA and type C consisting of $70 \% \mathrm{LC}$ and $30 \%$ PMMA, and finally the type D corresponding to $52 \% \mathrm{LC}$ and $48 \%$ PVB. On their basis, the developers made PDLC films of various thicknesses $h$, the list of which and the used marking are given in Table 2. The samples based on PMMA had a noticeable cloudiness, whereas the PVB-based samples were absolutely transparent. Such a difference in the transparency of the produced films can be related to different sizes of LC droplets in them. Probably the PVB film has a smaller size of LC droplets than visible band optic radiation wavelength, whereas in the PMMA film the LC droplets size is commensurable with or larger than the visible band optic radiation wavelength.

Table 2. Parameters of PDLC films.

\begin{tabular}{|l|l|c|c|}
\hline $\begin{array}{c}\text { Sample } \\
\text { commerce } \\
\text { title }\end{array}$ & $\begin{array}{c}\text { Polymer } \\
\text { type }\end{array}$ & $\begin{array}{c}\text { Ratio } \\
\text { LC:polymer } \\
\text { (in weight } \\
\text { fractions) }\end{array}$ & $\begin{array}{c}\text { Thickness } \\
\text { of films } \\
(\mu \mathrm{m})\end{array}$ \\
\hline A6 & PMMA & $11: 9$ & 6 \\
\hline A11 & PMMA & $11: 9$ & 11 \\
\hline B16 & PMMA & $13: 7$ & 16 \\
\hline C17 & PMMA & $14: 6$ & 17 \\
\hline C24 & PMMA & $14: 6$ & 24 \\
\hline D4 & PVB & $13: 12$ & 4 \\
\hline D5 & PVB & $13: 12$ & 5 \\
\hline D9 & PVB & $13: 12$ & 9 \\
\hline
\end{tabular}

\section{Method of measurements for electrooptical parameters of PDLC films}

The developers enable to study the transparency of PVBbased liquid-crystal dispersions by using the setup, the scheme of which is shown in Fig. 1. It enables to measure optical characteristics of light-scattering and transparent objects within the range of wavelengths from 450 up to $750 \mathrm{~nm}$. It consists of two main parts. One is illuminant and another is registering. The illuminant part includes a monochromator 1 , pinhole aperture 2 located in the monochromator output plane, lens 3 and shutter 4 . The lens 3 forms a narrow light beam equal to 15 angular minutes which is reflected from the mirror 5 and passes through the object under study 6 placed horizontally. The shutter 4 modulates radiation falling on the object under investigation with the frequency $1000 \mathrm{~Hz}$. The registering part includes the lens 7 and diaphragm 8 with a changeable aperture enabling to change the receiving system viewing angle from 35 angular minutes to 4.6 angular degrees. The radiation which passed through the diaphragm 8 hits the photomultiplier 9 , the signal of which is amplified with a narrow-band amplifier 10 and is registered with the digital voltmeter 11 . The test measurements showed that this facility with the corresponding illuminator capability, noise level, and linearity of the receiving system provides measuring the sample transmission coefficients within the wide range from 1 down to $10^{-6}$ with measurement errors less than $4 \%$.

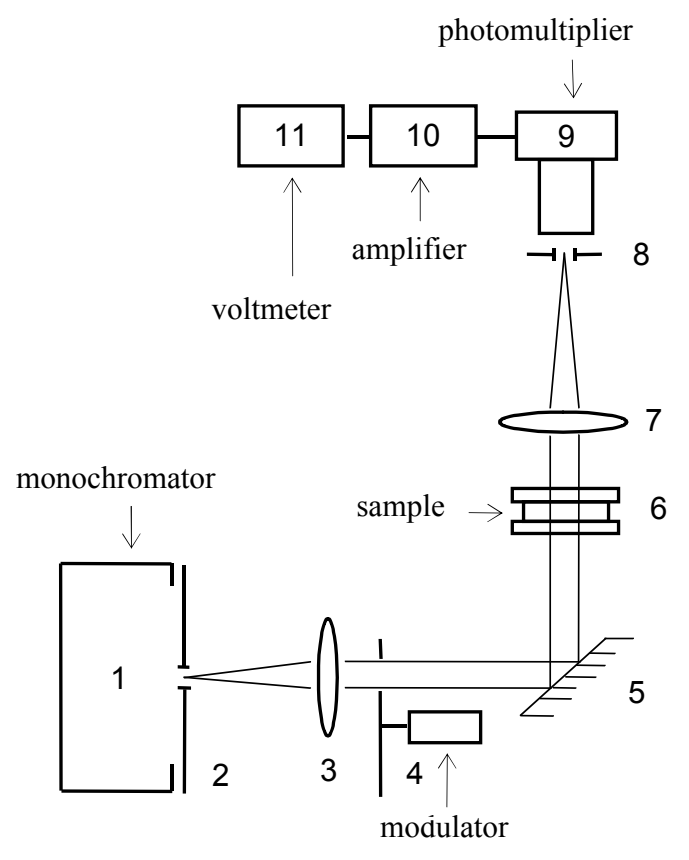

Fig. 1. Scheme of the experimental facility to measure the transmission coefficient of light-scattering objects. 


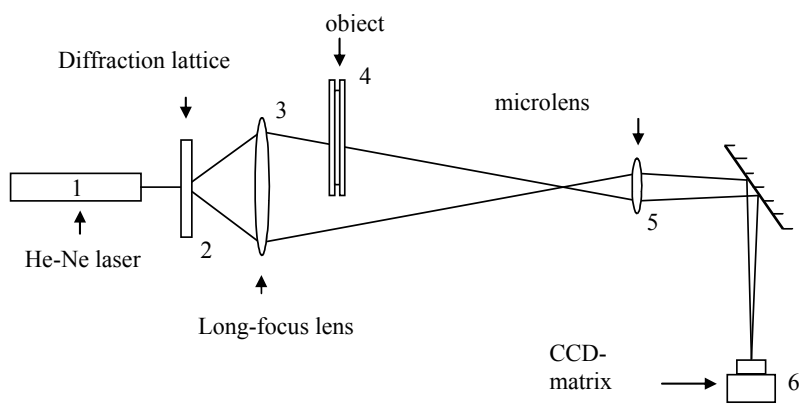

Fig. 2. Scheme of the experimental facility to measure the refraction index of dispersed media.

The measurement method and facility setup to determine the phase shift in the layers of polymer with dispersed crystal have been chosen coming from the specificity of the object under examination. The following peculiarities should be taken into account: the necessity to measure diffractive indices of nonhomogeneous media such as LC dispersions and the fact that thin films are located between transparent glass plates with different control voltages applied to these plates. As the measurement method that will meet the above-mentioned requirements, the authors chose the procedure based on the use of double-beam interferometer. The scheme of this facility is shown in Fig. 2. It consists of the helium-neon laser 1 , diffraction lattice 2, long-focus lens 3 , microlens 5 and registering device 6 . The diffraction lattice is used to split laser beam and to form two noncollinear coherent light beams, into one of which the investigated object 4 is placed. The long-focus lens 3 converges two coherent light beams. In beam crossing area owing to their coherence the interference pattern is formed. This pattern is magnified with the help of the microlens 5 and is registered with a CCD camera 6 connected with PC. The light wave phase shift is determined by the position of interference pattern bands in CCD matrix plane.

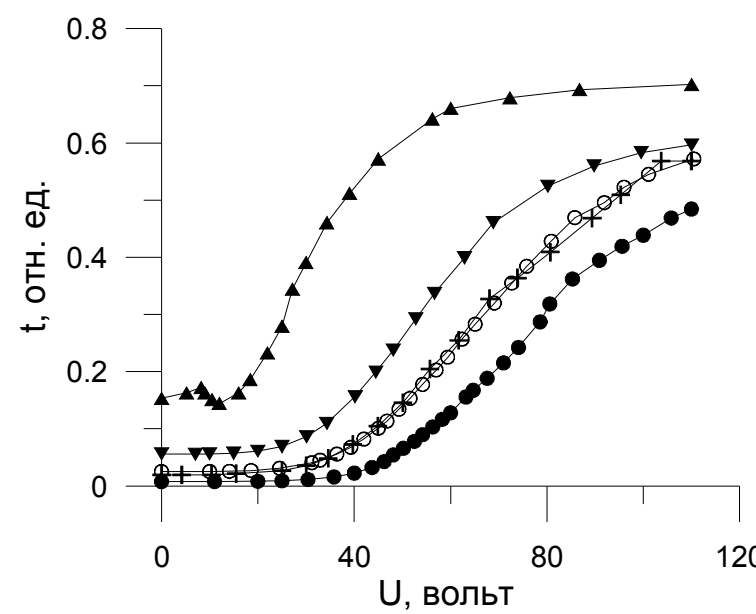

\section{Results and discussion}

The measurement results for the transmission coefficient $t$ and phase shift $\Delta \varphi$, carried out for the above-mentioned samples from PMMA, are shown in Fig. 3. Phase shift $\Delta \varphi$ measurement results for PVB samples are given in Fig. 4.

From Fig. 3, one can see that the transparency of the investigated PMMA PDLC films is primarily determined by their thickness $h$. The most transparent is 6- $\mu$ m layer A6. 24- $\mu \mathrm{m}$ layer C24 is optically the densest. Transmission sensitivity to external electric field for the investigated films is also primarily determined by their thickness $h$. Particularly, with increase of the film thickness the threshold voltage is growing (the voltage at which transmission by $10 \%$ exceeds the minimal one).

The phase shift value for PMMA samples, as can be seen from Fig. 3, strongly depends on both the LC concentration in a film and its thickness. When all this is going on, 6- $\mu \mathrm{m}$ thin layer A6 has the best factors, in the process of formation of which the mixture LC-PMMA in ratio 11:9 was used. For this film, the phase shift $\Delta \varphi$ reaches 5 radians at the electric field voltage reaching approximately $10 \mathrm{~V} / \mu \mathrm{m}$.

For PVB samples, as can be seen from Fig. 4, the value of the phase shift strongly depends on its thickness. At the same time, the thinnest layer D4 with the thickness of $4 \mu \mathrm{m}$ is the best factor. For this film, the phase shift $\Delta \varphi$ reaches 4 radians at the electric field voltage of about $20 \mathrm{~V} / \mu \mathrm{m}$.

The most adequate parameters characterizing optical properties of LC dispersions are the transmission coefficient and phase shift of the layers with a unit thickness. Therefore, from experimental data the specific phase shift $\Delta \varphi_{1}$ and attenuation index $\mu$ were calculated. They are numerically equal to the phase shift and optical thickness for PDLC films of unit thickness. Calculation of $\Delta \varphi_{1}$ and $\mu$ was carried out using the following formulas

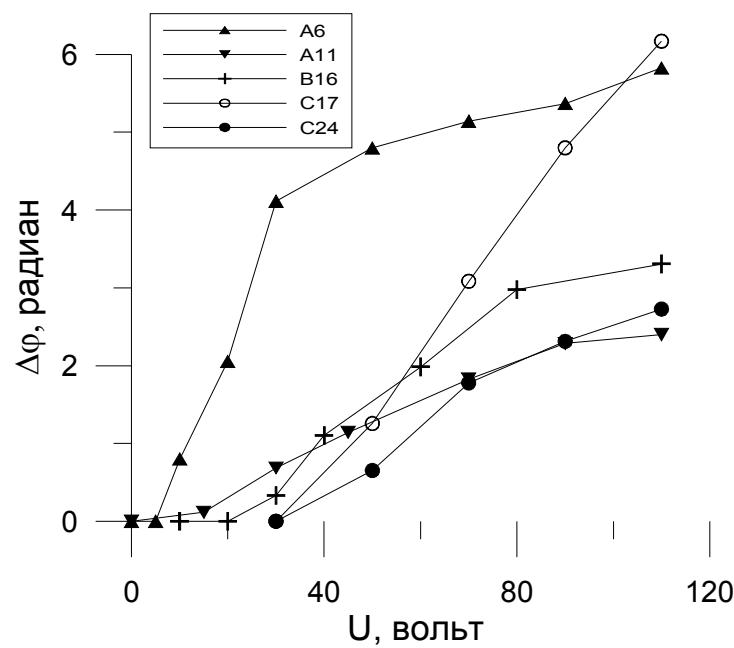

Fig. 3. Transmission $t$ and phase shift $\Delta \varphi$ of PMMA PDLC films at various applied voltages $U$. 


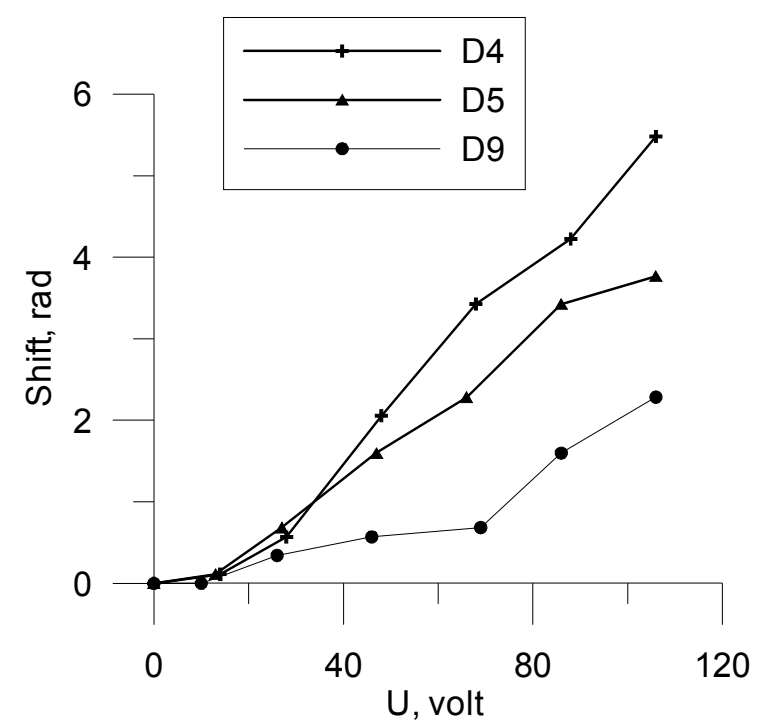

Fig. 4. Phase shift $\Delta \varphi$ in PVB PDLC films at various applied voltages $U$.

$\Delta \varphi_{1}=\Delta \varphi / h$,

and

$\mu=-\ln \left(t / t_{0}\right) / h$,

where $t_{0}$ is the transmission of glasses, between which PDLC layer was located (in our experiments the transmission $t_{0}$ was equal to 0.79 ).

Values $\Delta \varphi_{1}$ and $\mu$ for the investigated PMMA PDLC films and $\Delta \varphi_{1}$ for PVB PDLC films depending on the voltage of applied electric field are shown in Figs 5 and 6, respectively. From Fig. 5, one can see that the electrooptical parameters of PMMA PDLC films essentially depend on the method for making PDLC films, namely, on the solution evaporation rate in the process of drying the PDLC film. In this case, the solution evaporation rate, when the PDLC film is in the process of drying, somehow affects on the attenuation index $\mu$. Particularly, the $6-\mu \mathrm{m}$ film A6 is the most transparent, since in the process of formation the solution evaporation rate was maximal, and the $24-\mu \mathrm{m}$ film A6 is the least transparent, since in the process of formation the solution evaporation rate was minimal.

As it follows from Fig. 5, the increase of the LC fraction in LC-PMMA mixture from $55 \%$ to $70 \%$ has a comparatively low effect on the specific phase shift $\Delta \varphi_{1}$. At the same time, the value of $\Delta \varphi_{1}$ considerably depends on the solution evaporation rate. And namely, from the comparison of $\Delta \varphi_{1}$ values for films A6 and A11, including $\mathrm{C} 17$ and $\mathrm{C} 24$, it is obvious that the larger the value of $\Delta \varphi_{1}$ is, the higher is the rate of solution evaporation. In fairly thin films $\mathrm{A} 6$ and A11, the dependence of $\Delta \varphi_{1}$ is especially noticeable.

As it follows from Fig. 6, the properties of PVB PDLC films demonstrate a considerably low dependence on the solution evaporation rate. Fig. 6 shows that for PVB PDLC films the specific phase shift $\Delta \varphi_{1}$ linearly

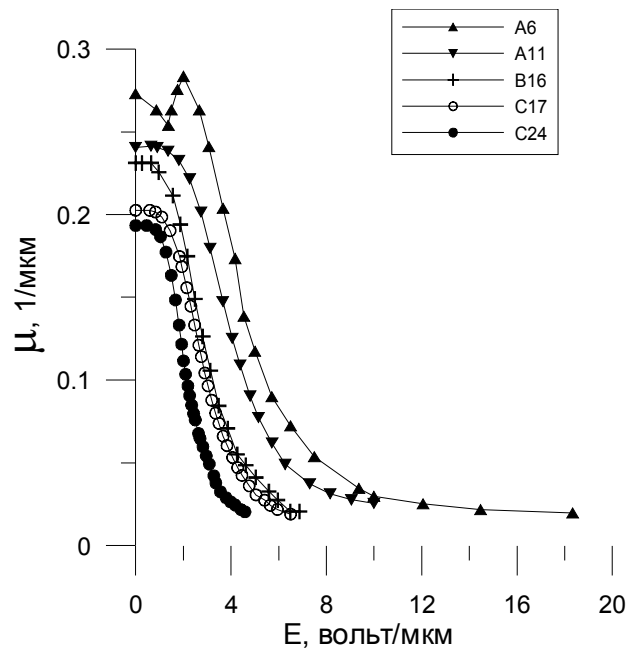

a)

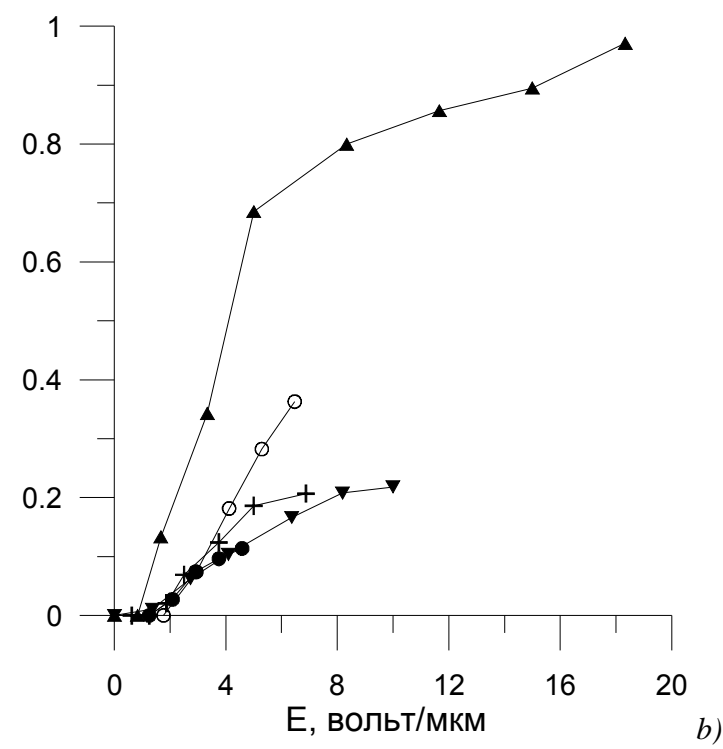

Fig. 5. Attenuation index $\mu$ and specific phase shift $\Delta \varphi_{1}$ of PMMA PDLC films at various applied electric fields $E$.

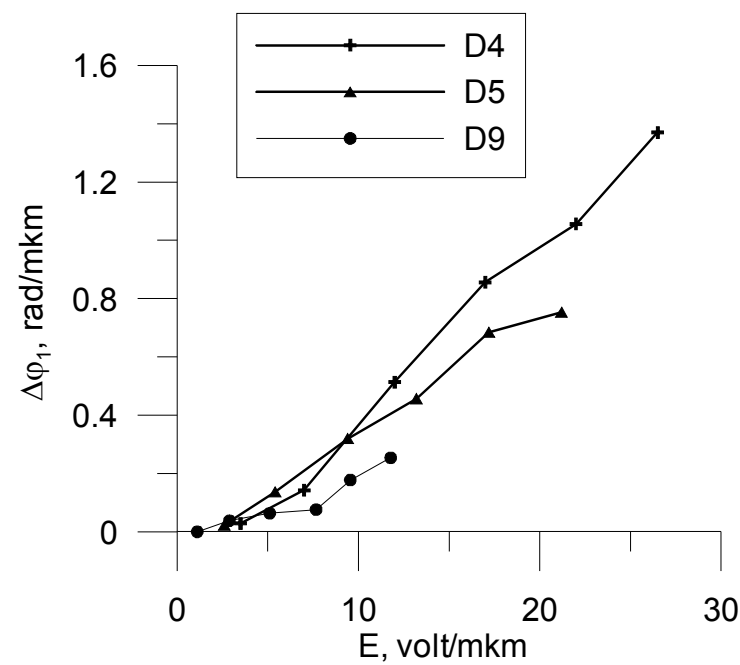

Fig. 6. Specific phase shift $\Delta \varphi_{1}$ of PVB PDLC films at various applied electric fields $E$. 
depends on the voltage of the applied field. The higher the voltage is, the thinner is the PDLC film. In other words, like to the case with PMMA PDLC films, the larger is the value of $\Delta \varphi_{1}$, the higher is the rate of solution evaporation. However, in case of PVB PDLC films this dependence is far less noticeable.

The data shown in Figs 3 to 6 have been obtained for linearly polarized incident radiation. To study the influence of the polarization state for incident radiation on electrooptical parameters of PDLC films, transmission and phase shift were examined at various orientation of the radiation polarization plane. The obtained results show that PDLC films transmission and phase shift are the same in case of changing the incident radiation polarization state, so they are polarizationindependent.

\section{Conclusion}

Experimental investigation of electrooptical parameters of PDLC films obtained by using the solution evaporation method has been carried out. The filmmaking procedure and the results of transmission and phase shift measurements are presented. It was shown that for PMMA PDLC films the solution evaporation rate is the main factor influencing their transparency and ability to change the light-wave phase. The higher solution evaporation rate is, the more transparent PDLC films are, and the stronger is the light-wave phase shift. Its value does not depend on the state of incident radiation polarization. For thin films, the phase shift can reach 5 radians at the voltages of the applied electric field of about $10 \mathrm{~V} / \mu \mathrm{m}$.

As it follows from the results of studies, the use of PVB as a polymer matrix enables one to obtain absolutely transparent (with no scattering of visible light) PDLC films. The value of the phase shift linearly depends on the voltage of applied electric field. It can reach 4 radians at the applied electric field voltage of about $20 \mathrm{~V} / \mu \mathrm{m}$.

\section{References}

1. F. Vita, A. Marino, V. Tkachenko, and G. Abbate, Visible and near-infrared characterization and modelling of nanosized holographic-polymerdispersed liquid crystal gratings // Phys. Rev. E 72, 011702-8 (2005).
2. I. Amimori, E.J. Eakin, J. Qi, G. Skaèej, S. Žumer, and P. Gregory, Surface-induced orientational order in stretched nanoscale-sized polymer dispersed liquid-crystal droplets // Phys. Rev. E 71, 031702-11 (2005).

3. R. Benmouna, V. Rachet, P. Le Barny, P. Feneyrou, U. Maschke, and X. Coqueret, FTIR and UV-Visible spectroscopy studies of thiolene/E7 composites showing nanodispersions: Curing kinetics and electro-optical properties // J. Polymer Eng. 26, p. 499-510 (2006).

4. L. McKenna, L.S. Miller, and I.R. Peterson, Polymer dispersed liquid crystal films for modulating infra-red radiation //Polymer 45, p. 6977-6984 (2004).

5. L.O. Dolgov and O.V. Yaroshchuk, Lightscattering liquid-crystal composites with reduced off-axis haze // J. SID 14, p. 657-662 (2006).

6. H. Ren, Y.-H. Lin, Y.-H. Fan, and S.T. Wu, Polarization-independent phase modulation using a polymer dispersed liquid crystal //Appl. Phys. Lett. 86, 141101-3 (2005).

7. H. Ren, Y.-H. Lin, Y.-H. Fan, and S.T. Wu, Tunable-focus microlens arrays using nanosized polymer-dispersed liquid crystal droplets // Optics Communs. 247, p. 101-106 (2005).

8. D.E. Luchetta, R. Karapinar, A. Manni, and F. Simoni, Phase-only modulation by nanosized polymer-dispersed liquid crystals // J. Appl. Phys. 91, p. 6060-6065 (2002).

9. V.P. Dick and V.A. Loiko, Optical phase shift by polymer dispersed liquid crystal films with fine droplets // J. Phys. D 37, p. 1834-1840 (2004).

10. L. Vicari, Electro-optic phase modulation by polymer dispersed liquid crystals // J. Appl. Phys. 81, p. 6612-6615 (1997).

11. P.S. Drzaic, Liquid Crystals Dispersions. World Scientific, Singapore, 1995.

12. F. Simoni, Nonlinear Optical Properties of Liquid Crystals and Polymer Dispersed Lliquid Crystals. World Scientific, Singapore, 1997.

13. V.G. Chigrinov, Liquid Crystal Devices: Physics and Applications. Artech House, Boston, London, 1999.

14. P.J. Collings, Liguid Crystals; Nature's Delicate Phase of Matter. Princeton University Press, Princeton, 2002. 